\title{
On Computation and Analysis of Topological Index-Based Invariants for Complex Coronoid Systems
}

\author{
Muhammad Aamer Rashid $\mathbb{D}^{1},{ }^{1}$ Sarfraz Ahmad $\left(\mathbb{D},{ }^{1}\right.$ Muhammad Kamran Siddiqui $\mathbb{D}^{1}$, \\ and Mohammed K. A. Kaabar $\mathbb{D}^{2,3,4}$ \\ ${ }^{1}$ Department of Mathematics, COMSATS University Islamabad, Lahore Campus, Lahore 54000, Pakistan \\ ${ }^{2}$ Gofa Campus, Near Gofa Industrial College and German Adebabay, Nifas Silk-Lafto, 26649, Addis Ababa, Ethiopia \\ ${ }^{3}$ Jabalia Campus, United Nations Relief and Works Agency (UNRWA) Palestinian Refugee Camp, \\ Gaza Strip Jabalya, State of Palestine \\ ${ }^{4}$ Institute of Mathematical Sciences, Faculty of Science, University of Malaya, Kuala Lumpur 50603, Malaysia \\ Correspondence should be addressed to Mohammed K. A. Kaabar; mohammed.kaabar@wsu.edu
}

Received 8 May 2021; Accepted 22 September 2021; Published 18 October 2021

Academic Editor: Miaomiao Wang

Copyright (c) 2021 Muhammad Aamer Rashid et al. This is an open access article distributed under the Creative Commons Attribution License, which permits unrestricted use, distribution, and reproduction in any medium, provided the original work is properly cited.

\begin{abstract}
In chemical graph theory, benzenoid systems are interrogated as they exhibit the chemical compounds known as benzenoid hydrocarbons. Benzenoid schemes are circumscribed as planar connected finite graphs having no cut vertices wherein the entire internal sections are collaboratively congruent regular hexagon. The past couple of decennium has acknowledged an extravagant development regarding implementation of information theoretic framework in miscellaneous ramification of science, for instance, in social sciences, biological, physical, and engineering. Explicitly, this tremendous improvement has been outstanding in the field of soft computing, molecular biology, and information technology. The information theory, delineated by Claud Shannon, has no less importance when it was considered. Shannon put forwarded the apprehension of entropy to enumerate upper bounds in transmission rates in telephonic channels, in optical communication, and in wireless. The prestigious feature of entropy is that it entitles the amount of uncertainty in a system. The substantial participation of this paper is to explore characteristics of graph entropies and then keep moving forward to talk about the formation of coronoid polycyclic aromatic hydrocarbons. Likewise, we estimate entropies through precise topological indices established on degree of terminal nodes.
\end{abstract}

\section{Introduction}

Consider $\mathscr{D}=\left(\mathscr{D}_{V}, \mathscr{D}_{E}\right)$ be a graph containing $\mathscr{D}_{V}$ and $\mathscr{D}_{E}$ as the vertex set and the edge set of $\mathscr{D}$ correspondingly. The size and the order of $\mathscr{D}$ are expressed by $m$ and $n$ correspondingly, and $\widehat{I}(a)$ is characterized as the degree of any vertex $a$. Topological descriptors of a chemical structure are molecular descriptors. In QSPR/QSAR analyses, miscellaneous molecular descriptor is operated to correlate different biological and physico-chemical activities. In this study, we will talk about some degree-based indices.

To determine the unpredictability of a scheme, entropy is used [1]. This consideration was grown for analyzing the fundamental information of graphs. Laterally, it was employed substantially in graphs and chemical networks.
The graph entropy [2] consideration demonstrated on the denominations of vertex orbits. Utilization of graph entropy is interdisciplinary [3].

In the literature, diverse graph entropies are estimated by means of degree of vertex, order of the graphs, eccentricity of the vertices, and characteristic polynomials $[4,5]$. Over the past few years, graph entropies are estimated which are established on matchings, independent sets, and degree of vertices [6]. Mowshowits and Dehmer talked about few relations between the complexity of graphs and Hosoya entropy. We postulated that the presented degree-based entropy can be employed to assess network diversity. Equivalent entropy measures which are established on vertex-degrees to distinguish network diversity have been suggested by Tan and $\mathrm{Wu}[7]$. 
In chemical graph theory, benzenoid structures are interrogated [8] since they exhibit the chemical compounds known as benzenoid hydrocarbons. Benzenoid schemes are circumscribed as planar connected finite graphs having no cut vertices wherein the entire internal sections are collaboratively congruent regular hexagons.

In [9], interpretation of the entropy was put forwarded for the edge weighted graph $\mathscr{D}=\left(\mathscr{D}_{v} ; \mathscr{D}_{E} ; \psi(a z)\right)$, in which $\mathscr{D}_{V}, \mathscr{D}_{E}$, and $\psi(a z)$ symbolize the set of vertices, the set of edges, and the edge weight of the edge $(a z)$ in $\mathscr{D}$ correspondingly. Subsequently the entropy of the edge weighted graph is portrayed in the following equation. The relation between topological indices and corresponding entropy measures is presented in Table 1.

$$
E N T_{\psi}(\mathscr{D})=-\sum_{a^{\prime} z^{\prime} \in \mathscr{D}_{e}} \frac{\psi\left(a^{\prime} z^{\prime}\right)}{\sum_{a z \in \mathscr{D}_{E}} \psi(a z)} \log \left[\frac{\psi\left(a^{\prime} z^{\prime}\right)}{\sum_{a z \in \mathscr{D}_{E}} \psi(a z)}\right] .
$$

\section{Structure of Coronoid Polycyclic Aromatic Hydrocarbons}

Coronoids are derived by these benzenoid systems by removing some interior vertices or edges. This will create a different interior region enclosed by a polygon having greater than six edges. By using the abovementioned conditions, more than one hexagon can be trimmed from the originator benzenoid structure. Coronoid is a benzenoid with a hole. It may have more than one hole. Graphical illustration of coronoid and noncoronoid systems is provided in Figures 1(a) and 1(b). It is to be noted that Figure 1(b) is noncoronoid because some of its edges do not belong to any of its hexagons [14].

tCycloarenes are macrocyclic combined compounds constituted by circumferentially connected benzene loops that enclose a hole with inner-directed carbon-hydrogen bonds. As a consequence, cycloarenes are connected with a subclass of circulenes or coronoids. The background of cycloarenes traces back to 1987; meanwhile, the main example with 12 benzene rings, categorized as kekulene, was disclosed by Staab and Diederich [15]. There have been countless hypothetical investigations focusing on the magnetic tendency, vibrational rate of occurrences of cycloarenes, and aromaticity [16]. Kekulene and cyclo decakis benzene were the merely two substantial patterns accessible for analysis [17].

Afterwards, Kumar et al. [18] synthesized another model of cycloarene, with 14 benzene rings, specifically septulene. The synthesis of kekulene and septulene has kick started numerous theoretical and experimental studies on coronoids [19]. The study of coronoids is also gaining momentum due to their superaromaticity. Superaromaticity or macrocyclic aromaticity is described as an additional thermodynamic consolidation as a consequence of macrocyclic association in tremendous-ring molecules like kekulene, and it constitutes a little contribution of universal aromaticity.

Whole coronoids investigated until now are approximately superaromatic through constructive superaromatic stabilization energies (SSEs). Intriguingly, the extent of SSE oscillates between single-layered and several-layered species. SSEs for even-layered coronoids [9, 20-22] are high, while those for odd-layered ones [5, 23, 24] are pretty low [23]. This provides an impetus for a deeper study into the properties of coronoid systems and their relationship with the underlying molecular structures. This study might be applicable to various fields of nanotechnology. As an illustration, the eradication of a unique carbon atom with a graphite framework establishes a one-atom hole referred to as a Schottky defect [25]. Individual-wall nanocones, [26] grime platelets, [27], and extended graphite layers [24] may contain vacancy hole defects involving larger (multiatom) holes which can be studied by modeling them as coronoids [28]. Graphenes are nanosized polycyclic aromatic hydrocarbons with potential uses in the fabrication of organic electronic devices [29]. The origination of coronoids by demonstrating a cavity in nanographene might be an efficacious approach to regulate their electronic and optical properties without amendments to their exterior structures. The cavities, that make an integral part of the coronoids, act as prototypes for scheming and synthesizing novel nanomaterials of significance in nano and biotechnology and the incipient field of nano-medicines. They have also been used in the design and synthesis of distinct porous and mesoporous materials grounded on calixarenes and mesoporous silica for the sequestration and complexation of toxic nuclear waste and other environmental pollutants [30] Coronoid systems are also widely examined in the study of coronoid hydrocarbons.

It has been proved that it is possible to compute the total $\pi$-electron energy, the resonance energy, and the enumeration of coronoid hydrocarbons accurately using the knowledge of coronoid structures [20]. The conjugate graphtheoretical circuit theory, inspired by Clar's aromatic sextet, correlates to the description of diverse enclosed consolidated cycles existing in the polycyclic aromatic compounds $[31,32]$. This theory also provides combinatorial and graphtheoretical methods for efficient determination of the relative stabilities of coronoid structures, graphenes, cycloarenes, carbon nanotubes, and nanotori. For further information on the comprehensive research issued on coronoid systems by both Dias with coauthors and Cyvin with coauthors, refer to [21]. In comparison to the computationally intensive quantum chemical calculations, the graph-theoretical techniques are considerably more productive in obtaining the properties of coronoid systems. During a recent investigation, Aihara et al. [23] emphasized that the graph theory is not merely an extremely valuable mechanism in estimating topological resonance energies but additionally in uncovering significant challenges along the previous speculations of aromaticity. By employing graphtheoretical approaches, the investigation of coronoid networks has gained increased importance [22].

\section{Coronoid System $C_{1}[u, v, w, x]$}

In this fragment, we will take into consideration the single coronoid system. This system is also recognised as one hole benzenoid. It is extracted by eradicating few of the interior edges or vertices from the benzenoid system. In this 
TABLE 1: Degree-based topological indices together with their corresponding edge weight $\psi(a z)$ of the edge $a z$.

\begin{tabular}{lcc}
\hline Degree-based topological indices & Edge weight $\psi(a z)$ of the edge $a z$ & Entropies \\
\hline The randic index [10] & $(\widehat{I}(r) \times \widehat{I}(s))^{\alpha}, \alpha=1,-1,1 / 2,-1 / 2$ & The randic entropy [11] \\
The atom bond connectivity index [12] & $\sqrt{\widehat{I}(r)+\widehat{I}(s)-2 / \widehat{I}(r) \times \hat{I}(s)}$ & The atom bond connectivity entropy [11] \\
The geometric arithmetic index [13] & $2 \sqrt{\hat{I}(r) \times \hat{I}(s) / \hat{I}(r)+\widehat{I}(s) .}$ & The geometric arithmetic entropy [11] \\
\hline
\end{tabular}

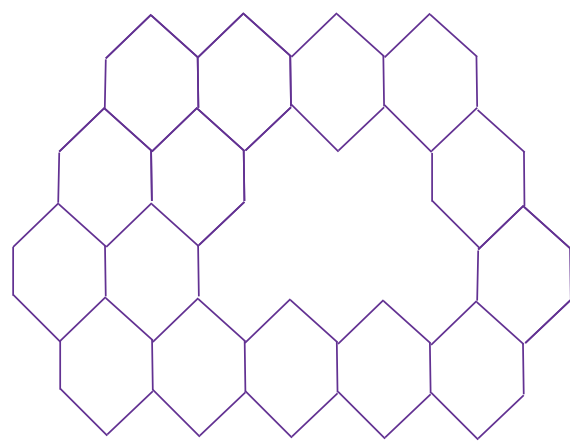

(a)

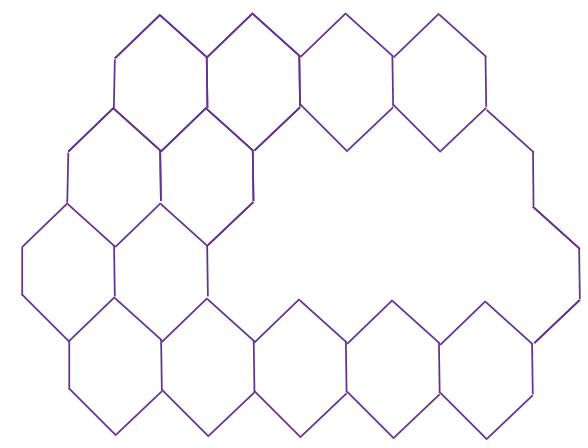

(b)

FIgURE 1: Coronoid and noncoronoid systems.

procedure, a hole is created within the system having a lowest size of two benzenes. The $x$-circumscribed basic coronoid system is defined as $C_{k}[u, v, w, x], k=1,2$, in which $u \geq 3$, $v, w \geq 1$, and $x \geq 1$. The coronoid structure fluctuates correspondingly to its parameters $u, v, w$, and $x$ bringing escalation to the particular cases. In [21,33], some particular cases are discussed, and these exclusive models are employed to prognosticate the resonance energy of aromatic molecules. In theoretical chemistry, these models are considered as ideal models to investigate conjugation circuits of $\pi$ electrons.

These special cases are used to predict the resonance energy of aromatic molecules and have attracted a great deal of interest in the field of theoretical chemistry as ideal models to explore conjugation circuits of $\pi$-electrons $[34,35]$. We will represent coronoid structure as $C_{1}[u, v, w, x]$ in which $u \geq 3$ and $1 \leq v \leq w \leq u$. Figure 2 illustrates $C_{1}[u, v, w, x]$ graphically.

Table 2 portrays the edge partition of $C_{1}[u, v, w, x]$ on the basis of degrees of terminal vertices of each edge.

\subsection{Results for Coronoid System $C_{1}[u, v, w, x]$}

3.1.1. Randic Entropy of $C_{1}[u, v, w, x]$. We enumerate the Randic index for $\alpha=1,-1,1 / 2,-1 / 2$ with the help of Tables 1 and 2 as follows:

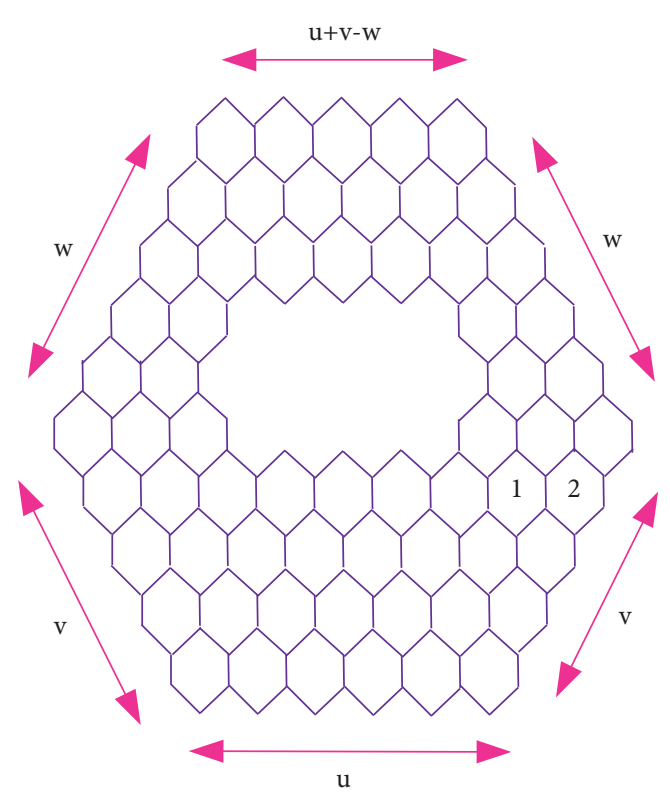

Figure 2: Coronoid system of $C_{1}[u, v, w, x][34]$.

$$
\begin{aligned}
R_{\alpha}\left(C_{1}[u, v, w, x]\right)= & (6) \times(4)^{\alpha}+(4 u+6 v+2 w+22) \times(6)^{\alpha} \\
& +\left((6 x+8) u+(9 x+12) v+(3 x+4) w+9 x^{2}+3 x-58\right) \times(9)^{\alpha} .
\end{aligned}
$$

Therefore, equation (1) with Tables 1 and 2 is in the form as follows: 
TABLE 2: Edge partition of the coronoid system $C_{1}[u, v, w, x]$ on the basis of degrees of terminal vertices of each edge.

\begin{tabular}{lcc}
\hline$(\widehat{I}(a), \widehat{I}(z))$ & Number of replication & Classes of edges \\
\hline$(2,2)$ & 6 & $\mathscr{D}_{E_{1}}$ \\
$(2,3)$ & $4 u+6 v+2 w+22$ & $\mathscr{D}_{E_{2}}$ \\
$(3,3)$ & $(6 x+8) u+(9 x+12) v+(3 x+4) w+9 x^{2}+3 x-58$ & $\mathscr{D}_{E_{3}}$ \\
\hline
\end{tabular}

$$
\begin{aligned}
\operatorname{ENT}_{R_{\alpha}}\left(C_{1}[u, v, w, x]\right)= & \log \left(R_{\alpha}\right)-\frac{1}{\left(R_{\alpha}\right)} \sum_{i=1}^{3} \sum_{a z \in \mathscr{D}_{E_{i}}}\left[(\widehat{I}(a) \times \widehat{I}(z))^{\alpha}\right] \log \left[(\widehat{I}(a) \times \widehat{I}(z))^{\alpha}\right], \\
\operatorname{ENT}_{R_{\alpha}}\left(C_{1}[u, v, w, x]\right)= & \log \left(R_{\alpha}\right)-\frac{6}{\left(R_{\alpha}\right)}\left(4^{\alpha}\right) \log \left[4^{\alpha}\right]-\frac{(4 u+6 v+2 w+22)}{\left(R_{\alpha}\right)}\left(6^{\alpha}\right) \log \left[6^{\alpha}\right] \\
& -\frac{(6 x+8) u+(9 x+12) v+(3 x+4) w+9 x^{2}+3 x-58}{\left(R_{\alpha}\right)}\left(9^{\alpha}\right) \log \left[9^{\alpha}\right],
\end{aligned}
$$

$\mathrm{ENT}_{R_{1}}\left(C_{1}[u, v, w, x]\right)=\log \left[(96+54 x) u+(144+81 x) v+(48+27 x) w+81 x^{2}+27 x-366\right]$

$$
\begin{aligned}
& 24 \log [4]
\end{aligned}
$$

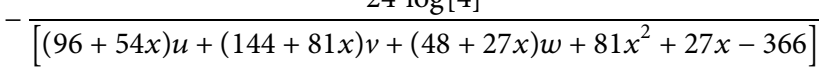

$$
\begin{aligned}
& 6(4 u+6 v+2 w+22) \log [6] \\
& -\overline{\left[(96+54 x) u+(144+81 x) v+(48+27 x) w+81 x^{2}+27 x-366\right]} \\
& -\frac{9\left((6 x+8) u+(9 x+12) v+(3 x+4) w+x^{2}+3 x-58\right) \log [9]}{\left[(96+54 x) u+(144+81 x) v+(48+27 x) w+81 x^{2}+27 x-366\right]} \\
& \operatorname{ENT}_{R_{1 / 2}}\left(C_{1}[u, v, w, x]\right)=\log \left[\begin{array}{c}
(4 \sqrt{6}+18 x+24) u+(6 \sqrt{6}+27 x+36) v \\
+(2 \sqrt{6}+9 x+12) w+27 x^{2}+9 x+22 \sqrt{6}-162
\end{array}\right] \\
& 12 \log [2] \\
& -\overline{\left[(4 \sqrt{6}+18 x+24) u+(6 \sqrt{6}+27 x+36) v+(2 \sqrt{6}+9 x+12) w+27 x^{2}+9 x+22 \sqrt{6}-162\right]} \\
& \sqrt{6}(4 u+6 v+2 w+22) \log [\sqrt{6}] \\
& -\frac{\left.(4 \sqrt{6}+18 x+24) u+(6 \sqrt{6}+27 x+36) v+(2 \sqrt{6}+9 x+12) w+27 x^{2}+9 x+22 \sqrt{6}-162\right]}{[0]} \\
& 3\left((6 x+8) u+(9 x+12) v+(3 x+4) w+x^{2}+3 x-58\right) \log [3] \\
& -\overline{\left[(4 \sqrt{6}+18 x+24) u+(6 \sqrt{6}+27 x+36) v+(2 \sqrt{6}+9 x+12) w+27 x^{2}+9 x+22 \sqrt{6}-162\right]}, \\
& \mathrm{ENT}_{R_{-1}}\left(C_{1}[u, v, w, x]\right)=\log \left[\left(\frac{14}{9}+\frac{2 x}{3}\right) u+\left(\frac{7}{3}+x\right) v+\left(\frac{7}{9}+\frac{x}{3}\right) w+x^{2}+\frac{x}{3}-\frac{23}{18}\right] \\
& +\frac{(3 / 2) \log [4]}{\left[((14 / 9)+(2 x / 3)) u+((7 / 3)+x) v+((7 / 9)+(x / 3)) w+x^{2}+(x / 3)-(23 / 18)\right]} \\
& +\frac{(1 / 6)(4 u+6 v+2 w+22) \log [6]}{\left[((14 / 9)+(2 x / 3)) u+((7 / 3)+x) v+((7 / 9)+(x / 3)) w+x^{2}+(x / 3)-(23 / 18)\right]} \\
& +\frac{(1 / 9)\left((6 x+8) u+(9 x+12) v+(3 x+4) w+x^{2}+3 x-58\right) \log [9]}{\left[((14 / 9)+(2 x / 3)) u+((7 / 3)+x) v+((7 / 9)+(x / 3)) w+x^{2}+(x / 3)-(23 / 18)\right]},
\end{aligned}
$$

$$
\begin{aligned}
\operatorname{ENT}_{R_{(-1 / 2)}}\left(C_{1}[u, v, w, x]\right)= & \log \left[\left(\frac{4}{\sqrt{6}}+2 x+\frac{8}{3}\right) u+(\sqrt{6}+3 x+4) v+\left(\frac{2}{\sqrt{6}}+x+\frac{4}{3}\right) w+3 x^{2}+x+\frac{22}{\sqrt{6}}-\frac{49}{3}\right] \\
& +\frac{(3 / 2) \log [2]}{\left[((4 / \sqrt{6})+2 x+(8 / 3)) u+(\sqrt{6}+3 x+4) v+((2 / \sqrt{6})+x+(4 / 3)) w+3 x^{2}+x+(22 / \sqrt{6})-(49 / 3)\right]} \\
& +\frac{(1 / \sqrt{6})(4 u+6 v+2 w+22) \log [\sqrt{6}]}{\left[((4 / \sqrt{6})+2 x+(8 / 3)) u+(\sqrt{6}+3 x+4) v+((2 / \sqrt{6})+x+(4 / 3)) w+3 x^{2}+x+(22 / \sqrt{6})-(49 / 3)\right]} \\
& +\frac{(1 / 3)\left((6 x+8) u+(9 x+12) v+(3 x+4) w+x^{2}+3 x-58\right) \log [3]}{\left[((4 / \sqrt{6})+2 x+(8 / 3)) u+(\sqrt{6}+3 x+4) v+((2 / \sqrt{6})+x+(4 / 3)) w+3 x^{2}+x+(22 / \sqrt{6})-(49 / 3)\right]} .
\end{aligned}
$$


3.1.2. The Atom Bond Connectivity Entropy of $C_{1}[u, v, w, x]$. Simple calculations with Tables 1 and 2 yield the atom bond connectivity index as follows:

$$
\begin{aligned}
A B C\left(C_{1}[u, v, w, x]\right)= & \left(2 \sqrt{2}+4 x+\frac{16}{3}\right) u \\
& +(3 \sqrt{2}+6 x+8) v+\left(\sqrt{2}+2 x+\frac{8}{3}\right) w \\
& +6 x^{2}+2 x+14 \sqrt{2}-\frac{116}{3} .
\end{aligned}
$$

Therefore, equation (1) with Tables 1 and 2 is in the form as follows:

$$
\begin{aligned}
& \operatorname{ENT}_{A B C}\left(C_{1}[u, v, w, x]\right)=\log (A B C)-\frac{1}{(A B C)} \sum_{i=1}^{3} \sum_{a z \in \mathscr{D}_{E_{i}}}\left[\sqrt{\frac{\widehat{I}(a)+\widehat{I}(z)-2}{\widehat{I}(a) \times \widehat{I}(z)}}\right] \log \left[\sqrt{\frac{\widehat{I}(a)+\widehat{I}(z)-2}{\widehat{I}(a) \times \widehat{I}(z)}}\right] \\
& \operatorname{ENT}_{A B C}\left(C_{1}[u, v, w, x]\right)=\log \left[\left(2 \sqrt{2}+4 x+\frac{16}{3}\right) u+(3 \sqrt{2}+6 x+8) v+\left(\sqrt{2}+2 x+\frac{8}{3}\right) w+6 x^{2}+2 x+14 \sqrt{2}-\frac{116}{3}\right] \\
& -\frac{6[1 / \sqrt{2}] \log [1 / \sqrt{2}]}{\left[(2 \sqrt{2}+4 x+(16 / 3)) u+(3 \sqrt{2}+6 x+8) v+(\sqrt{2}+2 x+(8 / 3)) w+6 x^{2}+2 x+14 \sqrt{2}-(116 / 3)\right]} \\
& -\frac{(4 u+6 v+2 w+22)[1 / \sqrt{2}] \log [1 / \sqrt{2}]}{\left[(2 \sqrt{2}+4 x+(16 / 3)) u+(3 \sqrt{2}+6 x+8) v+(\sqrt{2}+2 x+(8 / 3)) w+6 x^{2}+2 x+14 \sqrt{2}-(116 / 3)\right]} \\
& \left((6 x+8) u+(9 x+12) v+(3 x+4) w+x^{2}+3 x-58\right)[2 / 3] \log [2 / 3] \\
& -\frac{\left.(2 \sqrt{2}+4 x+(16 / 3)) u+(3 \sqrt{2}+6 x+8) v+(\sqrt{2}+2 x+(8 / 3)) w+6 x^{2}+2 x+14 \sqrt{2}-(116 / 3)\right]}{}
\end{aligned}
$$

3.1.3. The Geometric Arithmetic Entropy $C_{1}[u, v, w, x]$. Simple calculations with Tables 1 and 2 yield the geometric arithmetic index as follows:

$$
\begin{aligned}
\mathrm{GA}(G)= & \left(\frac{8 \sqrt{6}}{5}+6 x+8\right) u+\left(\frac{12 \sqrt{6}}{5}+9 x+12\right) v \\
& +\left(\frac{4 \sqrt{6}}{5}+3 x+4\right) w+9 x^{2}+3 x-52+\frac{44 \sqrt{6}}{5}
\end{aligned}
$$

$$
\begin{aligned}
\mathrm{ENT}_{\mathrm{GA}}\left(C_{1}[u, v, w, x]\right)= & \log (\mathrm{GA})-\frac{1}{(\mathrm{GA})} \sum_{i=1}^{3} \sum_{a z \in \mathscr{D}_{E_{i}}}\left[\frac{2 \sqrt{\widehat{I}(a) \times \widehat{I}(z)}}{\widehat{I}(a)+\widehat{I}(z)}\right] \log \left[\frac{2 \sqrt{\widehat{I}(a) \times \widehat{I}(z)}}{\widehat{I}(a)+\widehat{I}(z)}\right], \\
\mathrm{ENT}_{\mathrm{GA}}\left(C_{1}[u, v, w, x]\right)= & \log \left(\left(\frac{8 \sqrt{6}}{5}+6 x+8\right) u+\left(\frac{12 \sqrt{6}}{5}+9 x+12\right) v+\left(\frac{4 \sqrt{6}}{5}+3 x+4\right) w+9 x^{2}+3 x-52+\frac{44 \sqrt{6}}{5}\right) \\
& -\frac{(4 u+6 v+2 w+22)[2 \sqrt{6} / 5] \log [2 \sqrt{6} / 5]}{\left(((8 \sqrt{6} / 5)+6 x+8) u+((12 \sqrt{6} / 5)+9 x+12) v+((4 \sqrt{6} / 5)+3 x+4) w+9 x^{2}+3 x-52+(44 \sqrt{6} / 5)\right)} .
\end{aligned}
$$




\section{Coronoid System $C_{1}[u, v, w, x]$}

The coronoid system of $\mathscr{D}$ is delineated as $C_{2}[n, p, q, r]$, in which $x \geq 1, u \geq 3$, and $1 \leq v<w \leq u$. Figure 3 shows the coronoid system of $C_{2}[u, v, w, x][34,35]$.

The approach we will use here is to form the partitions of the edges of $C_{2}[u, v, w, x]$ of terminal vertices of each edge (see Table 3).
4.1. Results for Coronoid System $C_{2}[u, v, w, x]$

4.1.1. Randic Entropy of $C_{2}[u, v, w, x]$. We enumerate the Randic index for $\alpha=1,-1,1 / 2,-1 / 2$ with the help of Tables 3 and 1 as follows:

$$
\begin{aligned}
R_{\alpha}\left(C_{2}[u, v, w, x]\right)= & (6) \times(4)^{\alpha}+(4 u+4 v+4 w+24) \times(6)^{\alpha} \\
& +\left((6 u+6 v+6 w) x+9 x^{2}-9 x-40\right) \times(9)^{\alpha} .
\end{aligned}
$$

As a consequence, equation (1) with Tables 1 and 3 is embodied in the form as follows:

$$
\begin{aligned}
& \operatorname{ENT}_{R_{\alpha}}\left(C_{2}[u, v, w, x]\right)=\log \left(R_{\alpha}\right)-\frac{1}{\left(R_{\alpha}\right)} \sum_{i=1}^{3} \sum_{a z \in \mathscr{D}_{E_{i}}}\left[(\widehat{I}(a) \times \widehat{I}(z))^{\alpha}\right] \log \left[(\widehat{I}(a) \times \widehat{I}(z))^{\alpha}\right] \\
& \operatorname{ENT}_{R_{1}}\left(C_{2}[u, v, w, x]\right)=\log \left((24+54 x)(u+v+w)+81 x^{2}-81 x-192\right) \\
& 24 \log [4] \\
& -\overline{\left((24+54 x)(u+v+w)+81 x^{2}-81 x-192\right)} \\
& -\frac{6(4 u+4 v+4 w+24) \log [6]}{\left((24+54 x)(u+v+w)+81 x^{2}-81 x-192\right)}-\frac{9\left((6 u+6 v+6 w) r+9 x^{2}-9 x-40\right) \log [9]}{\left((24+54 x)(u+v+w)+81 x^{2}-81 x-192\right)}, \\
& \operatorname{ENT}_{R_{-1}}\left(C_{2}[u, v, w, x]\right)=\log \left(\frac{2}{3}(1+x)(u+v+w) x^{2}-x+\frac{19}{18}\right)+\frac{(3 / 2) \log [4]}{\left((2 / 3)(1+x)(u+v+w) x^{2}-x+(19 / 18)\right)} \\
& +\frac{(1 / 6)(4 u+4 v+4 w+24) \log [6]}{\left((2 / 3)(1+x)(u+v+w) x^{2}-x+(19 / 18)\right)}+\frac{(1 / 9)\left((6 u+6 v+6 w) x+9 x^{2}-9 x-40\right) \log [9]}{\left((2 / 3)(1+x)(u+v+w) x^{2}-x+(19 / 18)\right)}, \\
& \operatorname{ENT}_{R_{1 / 2}}\left(K_{2}[n, p, q, r]\right)=\log \left((4 \sqrt{6}+18)(n+p+q)+27 r^{2}-27 r+24 \sqrt{6}-108\right) \\
& 12 \log [2] \\
& -\overline{\left((4 \sqrt{6}+18)(n+p+q)+27 r^{2}-27 r+24 \sqrt{6}-108\right)} \\
& -\frac{\sqrt{6}(4 u+4 v+4 w+24) \log [\sqrt{6}]}{\left((4 \sqrt{6}+18)(n+p+q)+27 r^{2}-27 r+24 \sqrt{6}-108\right)} \\
& -\frac{3\left((6 u+6 v+6 w) x+9 x^{2}-9 x-40\right) \log [3]}{\left((4 \sqrt{6}+18)(n+p+q)+27 r^{2}-27 r+24 \sqrt{6}-108\right)},
\end{aligned}
$$

$\operatorname{ENT}_{R_{(-1 / 2)}}\left(C_{2}[u, v, w, x]\right)=\log \left(\left(\frac{4}{\sqrt{6}}+2 x\right)(u+v+w)+3 x^{2}-3 x+4 \sqrt{6}-\frac{31}{3}\right)$

$$
\begin{aligned}
& +\frac{(3 / 2) \log [2]}{\left(((4 / \sqrt{6})+2 x)(u+v+w)+3 x^{2}-3 x+4 \sqrt{6}-(31 / 3)\right)} \\
& +\frac{\sqrt{6}(4 u+4 v+4 w+24) \log [\sqrt{6}]}{(((4 / \sqrt{6})+2 x))(u+v+w)+3 x^{2}-3 x+4 \sqrt{6}-(31 / 3)} \\
& +\frac{(1 / 3)\left((6 u+6 v+6 w) x+9 x^{2}-9 x-40\right) \log [3]}{\left(((4 / \sqrt{6})+2 x)(u+v+w) t+n 3 q x^{2} h-{ }_{3} x x 7+C 4 ; \sqrt{6}-(31 / 3)\right)}
\end{aligned}
$$




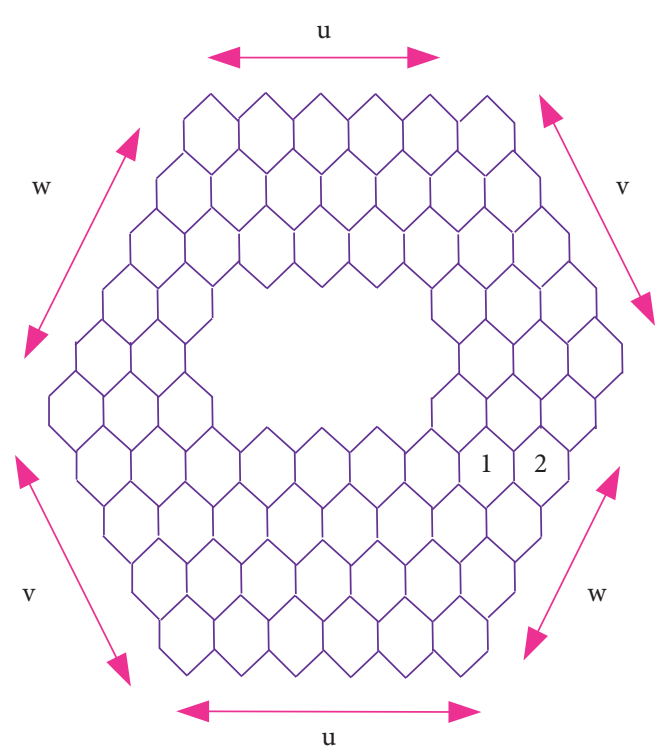

FIGURE 3: Coronoid system of $C_{2}[u, v, w, x][35]$.

TABLE 3: Edge partition of $C_{2}[u, v, w, x]$ established on degrees of terminal vertices of each edge.

\begin{tabular}{lcc}
\hline$(\widehat{I}(a), \widehat{I}(z))$ & Number of replication & Classes of edges \\
\hline$(2,2)$ & 6 & $\mathscr{G}_{E_{1}}$ \\
$(2,3)$ & $4 u+4 v+4 w+24$ & $\mathscr{G}_{E_{2}}$ \\
$(3,3)$ & $(6 u+6 v+6 w) x+9 x^{2}-9 x-40$ & $\mathscr{G}_{E_{3}}$ \\
\hline
\end{tabular}

4.1.2. The Atom Bond Connectivity Entropy of $C_{2}[u, v, w, x]$. Simple calculations with Tables 1 and 3 yield the atom bond connectivity index as follows:

$$
\begin{aligned}
A B C\left(C_{2}[u, v, w, x]\right)= & (2 \sqrt{2}+4 x)(u+v+w)+6 x^{2} \\
& -6 x+5 \sqrt{2}-\frac{80}{3}
\end{aligned}
$$

Therefore, equation (1) with Tables 1 and 3 is in the form as follows:

$$
\begin{aligned}
\operatorname{ENT}_{A B C}\left(C_{2}[u, v, w, x]\right)= & \log (A B C)-\frac{1}{(A B C)} \sum_{i=1}^{3} \sum_{a z \in \mathscr{D}_{E_{i}}}\left[\sqrt{\frac{\widehat{I}(a)+\widehat{I}(z)-2}{\widehat{I}(a) \times \widehat{I}(z)}}\right] \log \left[\sqrt{\left.\frac{\hat{I}(a)+\widehat{I}(z)-2}{\widehat{I}(a) \times \widehat{I}(z)}\right]}\right. \\
\operatorname{ENT}_{A B C}\left(C_{2}[u, v, w, x]\right)= & \log (A B C)-\frac{[6 / \sqrt{2}]}{(A B C)} \log \left[\frac{1}{\sqrt{2}}\right]-\frac{(4 u+4 v+4 w+24)}{(A B C)}\left[\frac{1}{\sqrt{2}}\right] \log \left[\frac{1}{\sqrt{2}}\right] \\
& -\frac{(6 u+6 v+6 w) x+9 x^{2}-9 x-40}{(A B C)}\left[\frac{2}{3}\right] \log \left[\frac{2}{3}\right] .
\end{aligned}
$$$$
\mathrm{GA}(G)=\left(\frac{8 \sqrt{6}}{5}+6 x\right)(u+v+w)+9 x^{2}-9 x-34+\frac{48 \sqrt{6}}{5}
$$

4.1.3. The Geometric Arithmetic Entropy of $C_{2}[u, v, w, x]$. Simple calculations with Tables 1 and 3 yield the geometric arithmetic index as follows: 
TABLE 4: Comparison of Randic entropies for $C_{1}[u, 1,1, x]$.

\begin{tabular}{lcccc}
\hline$[u, x]$ & $\mathrm{ENT}_{R_{1}}$ & $\mathrm{ENT}_{R_{-1}}$ & $\mathrm{ENT}_{R_{1 / 2}}$ & $\mathrm{ENT}_{R_{-1 / 2}}$ \\
\hline$[3,1]$ & 0.1132 & 1.8445 & 1.8542 & 2.1906 \\
{$[4,2]$} & 0.9964 & 2.1801 & 2.4296 & 2.0910 \\
{$[5,3]$} & 1.3732 & 2.4213 & 2.6157 & 2.2841 \\
{$[6,4]$} & 1.6202 & 2.6094 & 2.7684 & 2.4560 \\
{$[7,5]$} & 1.8065 & 2.7634 & 2.8980 & 2.7511 \\
{$[8,6]$} & 1.9571 & 2.8939 & 3.0305 & 2.8709 \\
{$[9,7]$} & 2.0839 & 3.0072 & 3.1701 & 3.0134 \\
{$[10,8]$} & 2.1938 & 3.1072 & 3.2793 & 3.1253 \\
{$[11,9]$} & 2.2907 & 3.1969 & 3.3802 & 3.2018 \\
{$[12,10]$} & 2.3776 & 3.2781 & & \\
\hline
\end{tabular}

TABLE 5: Comparison of $\mathrm{ENT}_{\mathrm{ABC}}$ and $\mathrm{ENT}_{\mathrm{GA}}$ entropies for $C_{1}[u, v, w, x]$.

\begin{tabular}{lcr}
\hline$[u, s]$ & $\mathrm{ENT}_{\mathrm{ABC}}$ & $\mathrm{ENT}_{\mathrm{GA}}$ \\
\hline$[3,1]$ & 1.8572 & 1.8360 \\
{$[4,2]$} & 2.203 & 2.2801 \\
{$[5,3]$} & 2.4412 & 2.5220 \\
{$[6,4]$} & 2.6269 & 2.7097 \\
{$[7,5]$} & 2.7893 & 2.8834 \\
{$[8,6]$} & 2.8987 & 2.9998 \\
{$[9,7]$} & 3.0111 & 3.0967 \\
{$[10,8]$} & 3.0806 & 3.1667 \\
{$[11,9]$} & 3.1697 & 3.2563 \\
{$[12,10]$} & 3.2605 & 3.3574 \\
\hline
\end{tabular}

Therefore, equation (1) with Tables 1 and 3 is in the form as follows:

$$
\begin{aligned}
\mathrm{ENT}_{\mathrm{GA}}\left(C_{2}[u, v, w, x]\right)= & \log (\mathrm{GA})-\frac{1}{(\mathrm{GA})} \sum_{i=1}^{3} \sum_{a z \in \mathscr{D}_{E_{i}}}\left[\frac{2 \sqrt{\widehat{I}(a) \times \widehat{I}(z)}}{\widehat{I}(a)+\widehat{I}(z)}\right] \log \left[\frac{2 \sqrt{\hat{I}(a) \times \widehat{I}(z)}}{\widehat{I}(a)+\widehat{I}(z)}\right], \\
\mathrm{ENT}_{\mathrm{GA}}\left(C_{2}[u, v, w, x]\right)= & \log \left(\left(\frac{8 \sqrt{6}}{5}+6 x\right)(u+v+w)+9 x^{2}-9 x-34+\frac{48 \sqrt{6}}{5}\right) \\
& -\frac{(4 u+4 v+4 w+24)[2 \sqrt{6} / 5] \log [2 \sqrt{6} / 5]}{\left(((8 \sqrt{6} / 5)+6 x)(u+v+w) t+n 9 q x^{2} h-{ }_{9} x x 7-C 34 ;+(48 \sqrt{6} / 5)\right)} .
\end{aligned}
$$

\section{Comparisons and Discussion for $C_{1}[u, v, w, x]$}

In QSPR/QSAR discussions, topological indices are applied to connect the biological functions of the anatomies with their corporeal properties like distortion, strain energy, stability, and melting point. These determinations can be accomplished by employing degree-based indices as these indices have clarity of decision and rapidity. In this research, we asseverated some degree-based entropies. We proposed a new approach to estimate the entropy by estimating its topological indices. The degree-based entropy can also be exerted to structural chemistry, ecological networks, biology, national security, social network, and so on. Additionally, to investigate structural symmetry and asymmetry in real networks, the values of entropies have a significant role.
Entropy is monotonically increasing function as in all situations. Here, we estimated mathematically all degree-based entropies for diverse values of $u$ and $x$ keeping $p=1$ and $q=1$ for $C_{1}[u, v, w, x]$. Besides, we construct Tables 4 and 5 for tiny values of $u, x, p=1$ and $q=1$ for degree-based entropy to numerical comparison for the structure of $C_{1}[u, v, w, x]$. Now, numerical comparison is represented in Tables 4 and 5. Also, the graphical comparison is depicted in Figures 4-6.

\section{Comparisons and Discussion for $C_{2}[u, v, w, x]$}

Multiple exploitations of complex networks deserted on the entropy corresponding with structural information are discussed. In [36-39], many methods were suggested to 


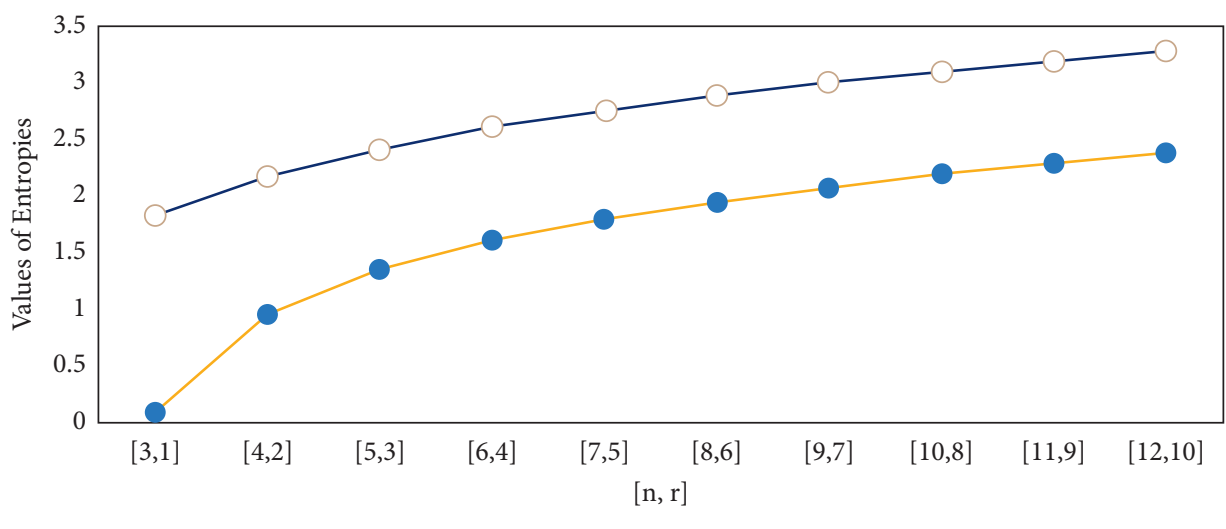

ENT-R1

$-\bigcirc$ ENT-R2

FIgURE 4: Comparison of Randic entropies for $\alpha=1$ and for $\alpha=-1$.

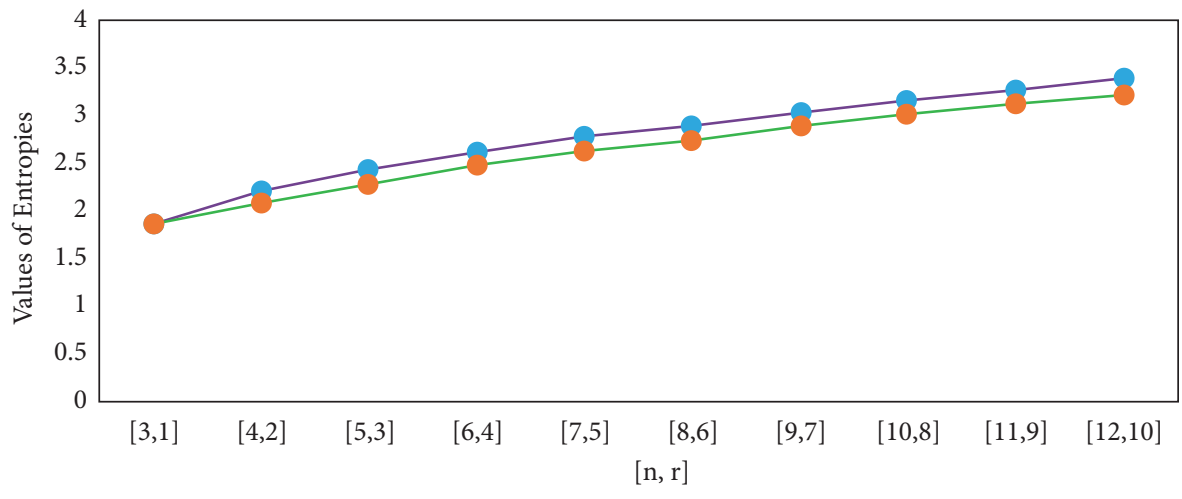

ENT-R3

ENT-R4

FIgURE 5: Comparison of Randic entropies for $\alpha=1 / 2$ and for $\alpha=-1 / 2$.

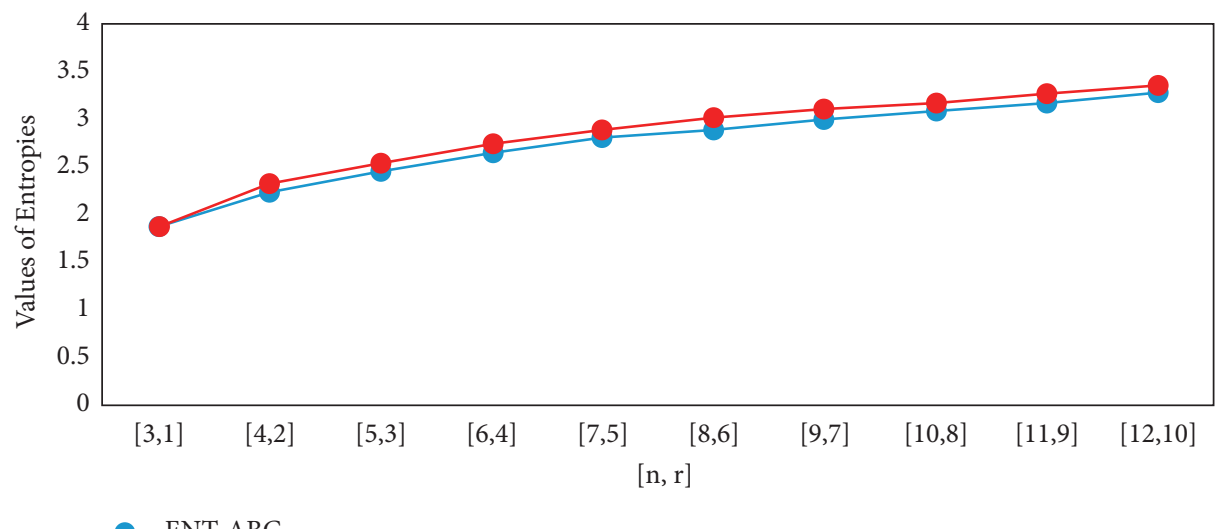

ENT-ABC

ENT-GA

FIgURE 6: Comparison of atom bond connectivity entropy and geometric arithmetic entropy.

check the structural complexity. However, the entropy approach is considered to be the most significant approach to discriminate the structural information of the complex networks. Besides, degree-based indices have theoretical role on account of having the capability of computing pharmaceutical properties. Therefore, we have listed mathematically some degree-based entropies for little considerations of parameters $u$ and $x$ keeping $v=1$ and $w=$ 2 for $C_{2}[u, v, w, x]$ as depicted in Tables 6 and 7. Also, the graphical comparison is depicted in Figures 7-9. 
TABLE 6: Comparison of Randic entropies for $C_{2}[u, v, w, x]$.

\begin{tabular}{lcccc}
\hline$[u, x]$ & $\mathrm{ENT}_{R_{1}}$ & $\mathrm{ENT}_{R_{-1}}$ & $\mathrm{ENT}_{R_{1 / 2}}$ & $\mathrm{ENT}_{R_{-1 / 2}}$ \\
\hline$[3,1]$ & 1.8378 & 1.8380 & 1.8433 & 2.1791 \\
{$[4,2]$} & 2.1715 & 2.2689 & 2.4195 & 2.5181 \\
{$[5,3]$} & 2.4143 & 2.5208 & 2.7070 & 2.6359 \\
{$[6,4]$} & 2.6032 & 2.7401 & 2.7608 & 2.7594 \\
{$[7,5]$} & 2.7579 & 2.9152 & 2.8912 & 2.8754 \\
{$[8,6]$} & 2.8890 & 3.0123 & 3.0045 & 3.078 \\
{$[9,7]$} & 3.0026 & 3.1307 & 3.1046 & 3.1661 \\
{$[10,8]$} & 3.1030 & 3.2114 & 3.1943 & 3.2469 \\
{$[11,9]$} & 3.1930 & 3.2915 & 3.2756 & 3.3213 \\
\hline 12,10$]$ & 3.2744 & 3.3732 & & \\
\hline
\end{tabular}

TABLE 7: Comparison of $\mathrm{ENT}_{\mathrm{ABC}}$ and $\mathrm{ENT}_{\mathrm{GA}}$ entropies for $C_{2}[u, v, w, x]$.

\begin{tabular}{lcr}
\hline$[u, x]$ & $\mathrm{ENT}_{\mathrm{ABC}}$ & $\mathrm{ENT}_{\mathrm{GA}}$ \\
\hline$[3,1]$ & 1.7596 & 1.8451 \\
{$[4,2]$} & 2.0944 & 2.1818 \\
{$[5,3]$} & 2.3402 & 2.4216 \\
{$[6,4]$} & 2.5347 & 2.6085 \\
{$[7,5]$} & 2.7122 & 2.7819 \\
{$[8,6]$} & 2.8249 & 2.8921 \\
{$[9,7]$} & 2.9497 & 3.0052 \\
{$[10,8]$} & 3.0583 & 3.1008 \\
{$[11,9]$} & 3.1512 & 3.1912 \\
{$[12,10]$} & 3.2531 & 3.2731 \\
\hline
\end{tabular}

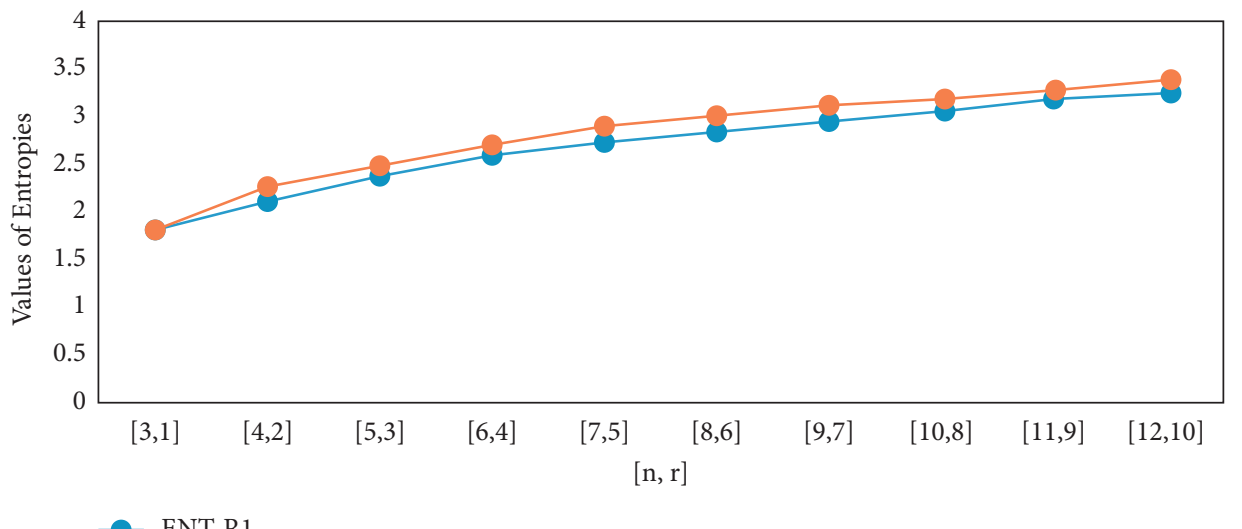

ENT-R1

ENT-R2

Figure 7: Comparison of Randic entropies for $\alpha=1$ and for $\alpha=-1$ for the coronoid system $C_{2}[u, v, w, x]$.

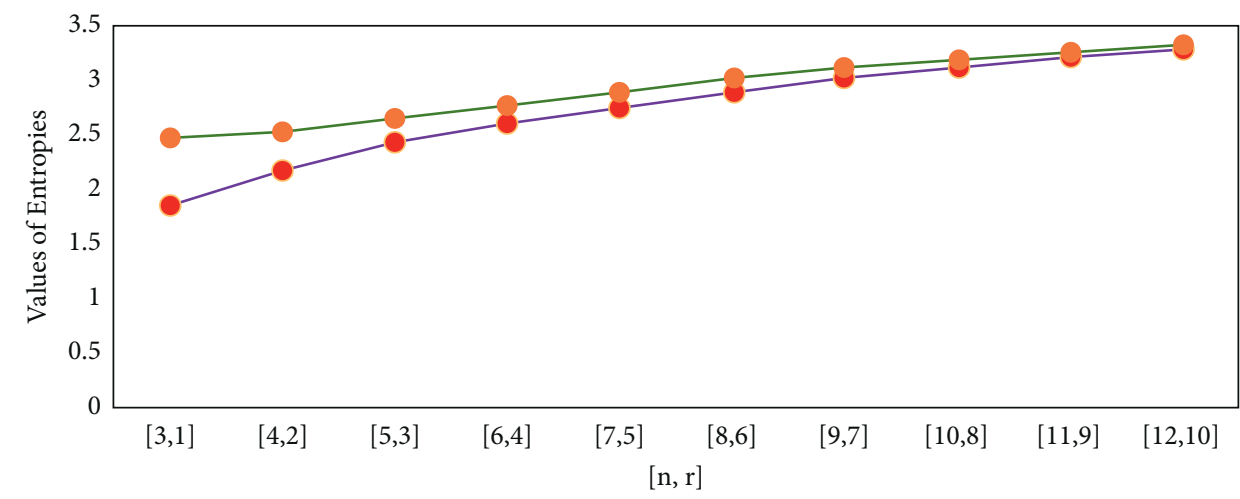

ENT-R3

ENT-R4

Figure 8: Comparison of Randic entropies for $\alpha=1 / 2$ and for $\alpha=-1 / 2$ for the coronoid system $C_{2}[u, v, w, x]$. 


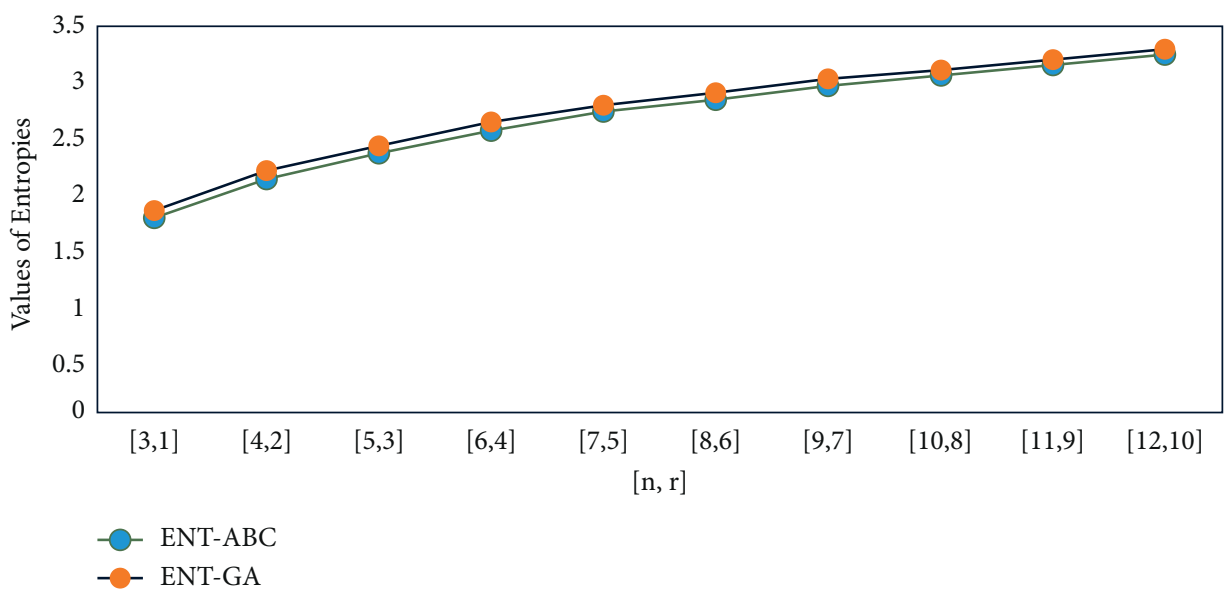

Figure 9: Comparison of Randic entropies for $\alpha=1 / 2$ and for $\alpha=-1 / 2$ for the coronoid system $C_{2}[u, v, w, x]$.

\section{Conclusion}

In this research, we have appropriated some degree-based indices for the characterization of the unambiguous graphtheoretical system of biochemical concern. We talked about topological indices, for instance, general Randic index, atomic bond connectivity index, and geometric arithmetic index for coronoid polycyclic aromatic hydrocarbons. Besides, we enumerated the corresponding entropies. The enumerated results link individual physico-chemical characteristics like distortion, stability, melting points, and strain energy of chemical compounds. The mathematical findings for these coronoid systems are helpful for the chemist to understand the biochemical applications of these coronoid systems $C_{1}[u, v, w, x]$ and $C_{2}[u, v, w, x]$.

\section{Data Availability}

No data were used to support this study.

\section{Conflicts of Interest}

The authors declare that there are no conflicts of interest.

\section{Authors' Contributions}

All authors contributed equally to this work.

\section{References}

[1] C. E. Shannon, "A mathematical theory of communication," Bell System Technical Journal, vol. 27, no. 3, pp. 379-423, 1948.

[2] N. Rashevsky, "Life, information theory, and topology," Bulletin of Mathematical Biophysics, vol. 17, no. 3, pp. 229235, 1955.

[3] R. E. Ulanowicz, "Quantitative methods for ecological network analysis," Computational Biology and Chemistry, vol. 28, no. 5-6, pp. 321-339, 2004.

[4] M. Dehmer, "Information processing in complex networks: graph entropy and information functionals," Applied Mathematics and Computation, vol. 201, no. 1-2, pp. 82-94, 2008.
[5] M. Dehmer and A. Mowshowitz, "A history of graph entropy measures," Information Sciences, vol. 181, no. 1, pp. 57-78, 2011.

[6] S. Manzoor, M. K. Siddiqui, and S. Ahmad, "On entropy measures of molecular graphs using topological indices," Arabian Journal of Chemistry, vol. 13, no. 8, pp. 6285-6298, 2020.

[7] Y. J. Tan and J. Wu, "Network structure entropy and its application to scale-free networks," Systems EngineeringTheory \& Practice, vol. 6, pp. 1-3, 2004.

[8] M. Randić, "Aromaticity of polycyclic conjugated hydrocarbons," Chemical Reviews, vol. 103, no. 9, pp. 3449-3605, 2003.

[9] Z. Chen, M. Dehmer, and Y. Shi, "A note on distance-based graph entropies," Entropy, vol. 16, no. 10, pp. 5416-5427, 2014.

[10] D. Amić, D. Bešlo, B. Lucić, S. Nikolić, and N. Trinajstić, "The vertex-connectivity index revisited," Journal of Chemical Information and Computer Sciences, vol. 38, no. 5, pp. 819822, 1998.

[11] S. Manzoor, M. K. Siddiqui, and S. Ahmad, "Degree-based entropy of molecular structure of hyaluronic acid-curcumin conjugates," The European Physical Journal Plus, vol. 136, no. 1, pp. 1-21, 2021.

[12] E. Estrada, L. Torres, L. Rodriguez, and I. Gutman, “An atombond connectivity index: modelling the enthalpy of formation of alkanes," Indian Journal of Chemistry, vol. 37A, pp. 849855, 1998.

[13] D. Vukičević and B. Furtula, "Topological index based on the ratios of geometrical and arithmetical means of end-vertex degrees of edges," Journal of Mathematical Chemistry, vol. 46, no. 4, pp. 1369-1376, 2009.

[14] Y. M. Chu, M. K. Siddiqui, S. Javed, L. Sherin, and F. Kausar, "On zagreb type molecular descriptors of ceria oxide and their applications," Journal of Cluster Science, pp. 1-10, 2021.

[15] H. A. Staab and F. Diederich, "Cycloarenes, a new class of aromatic compounds, I. Synthesis of kekulene," Chemische Berichte, vol. 116, no. 10, pp. 3487-3503, 1983.

[16] H. Miyoshi, S. Nobusue, A. Shimizu, and Y. Tobe, "Nonalternant non-benzenoid kekulenes: the birth of a new kekulene family," Chemical Society Reviews, vol. 44, no. 18, pp. 6560-6577, 2015.

[17] D. J. H. Funhoff and H. A. Staab, "Cyclo[d.e.d.e.e.d.e.d.e.e.] decakisbenzene, a New Cycloarene," Angewandte Chemie International Edition in English, vol. 25, no. 8, pp. 742-744, 1986. 
[18] B. Kumar, R. L. Viboh, M. C. Bonifacio et al., "Septulene: the heptagonal homologue of kekulene," Angewandte Chemie, vol. 124, no. 51, pp. 12967-12972, 2012.

[19] M. Randic, "Aromaticity and conjugation," Journal of the American Chemical Society, vol. 99, no. 2, pp. 444-450, 1977.

[20] J. Cioslowski, "Additive nodal increments for approximate calculation of the total ?-electron energy of benzenoid hydrocarbons," Theoretica Chimica Acta, vol. 68, no. 4, pp. 315-319, 1985.

[21] J. R. Dias, "Structure and electronic characteristics of coronoid polycyclic aromatic hydrocarbons as potential models of graphite layers with hole defects," The Journal of Physical Chemistry A, vol. 112, no. 47, pp. 12281-12292, 2008.

[22] J. R. Dias, "Handbook of polycyclic hydrocarbons," Part A: Benzenoid Hydrocarbons, John Wiley \& Sons, New Jersey, USA, 1987.

[23] J.-i. Aihara, M. Makino, T. Ishida, and J. R. Dias, "Analytical study of superaromaticity in cycloarenes and related coronoid hydrocarbons," The Journal of Physical Chemistry A, vol. 117, no. 22, pp. 4688-4697, 2013.

[24] J. R. Dias, "On the spectacular structural isomorphism between $\mathrm{CnHs}$ monoradical and $\mathrm{Cn}+\mathrm{sHs}+3$ diradical benzenoid hydrocarbons: from reactive intermediates to vacancy (hole) defects in graphite," The Journal of Physical Chemistry $A$, vol. 112, no. 14, pp. 3260-3274, 2008.

[25] F. Dietz, N. Tyutyulkov, G. Madjarova, and K. Müllen, "Is 2-D graphite an ultimate large hydrocarbon? II. Structure and energy spectra of polycyclic aromatic hydrocarbons with defects," The Journal of Physical Chemistry B, vol. 104, no. 8, pp. 1746-1761, 2000.

[26] A. T. Balaban, D. J. Klein, and X. Liu, "Graphitic cones," Carbon, vol. 32, no. 2, pp. 357-359, 1994.

[27] A. Giordana, A. Maranzana, G. Ghigo, M. Causà, and G. Tonachini, "Soot platelets and PAHs with an odd number of unsaturated carbon atoms and $\pi$ electrons: theoretical study of their spin properties and interaction with ozone," The Journal of Physical Chemistry A, vol. 112, no. 5, pp. 973-982, 2008.

[28] G. G. Hall, "Molecules with holes," Theoretica Chimica Acta, vol. 73, no. 5-6, pp. 425-435, 1988.

[29] J. Wu, W. Pisula, and K. Müllen, "Graphenes as potential material for electronics," Chemical Reviews, vol. 107, no. 3, pp. 718-747, 2007.

[30] T. Parsons-Moss, S. Jones, J. Wang et al., "Reduction of plutonium in acidic solutions by mesoporous carbons," Journal of Radioanalytical and Nuclear Chemistry, vol. 307, no. 3, pp. 2593-2601, 2016.

[31] Z. Raza, "The expected values of arithmetic bond connectivity and geometric indices in random phenylene chains," Heliyon, vol. 6, no. 7, Article ID e04479, 59 pages, 2020.

[32] Z. Raza and M. E. K. Sukaiti, "M-polynomial and degree based topological indices of some nanostructures," Symmetry, vol. 12, no. 5, pp. 831-841, 2020.

[33] M. Arockiaraj, J. Clement, and K. Balasubramanian, "Topological indices and their applications to circumcised donut benzenoid systems, kekulenes and drugs," Polycyclic Aromatic Compounds, vol. 40, no. 2, pp. 280-303, 2020.

[34] K. Julietraja and P. Venugopal, "Computation of degree-based topological descriptors using M-polynomial for coronoid systems," Polycyclic Aromatic Compounds, vol. 1, pp. 1-24, 2020.

[35] K. Julietraja, P. Venugopal, S. Prabhu, J.-B. Liu, and M. K. Siddiqui, "M-polynomial and degree-based molecular descriptors of certain classes of benzenoid systems," Polycyclic Aromatic Compounds, vol. 2, pp. 1-30, 2021.

[36] K. Anand and G. Bianconi, "Entropy measures for networks: toward an information theory of complex topologies," Physical Review E, vol. 80, no. 4, pp. 045102-045155, 2009.

[37] G. Bianconi, "The entropy of randomized network ensembles," Europhysics Letters, vol. 81, no. 2, pp. 28-45, 2008.

[38] M. Cai, R. Y. K. Du Hai-Feng, and W. Marcus, "A new network structure entropy based node difference and edge difference," Acta Physica Sinica, vol. 60, pp. 110-115, 2011.

[39] Y.-H. Xiao, W.-T. Wu, H. Wang, M. Xiong, and W. Wang, "Symmetry-based structure entropy of complex networks," Physica A: Statistical Mechanics and Its Applications, vol. 387, no. 11, pp. 2611-2619, 2008. 